\title{
Is quantitative fluorine-18 fluorodeoxyglucose PET image analysis the key to Identify cardiac sarcoidosis?
}

\author{
Richard C. Brunken, MD, FAHA, FACC, FASNC ${ }^{\mathrm{a}, \mathrm{b}}$ \\ a Department of Radiology, Cleveland Clinic Lerner College of Medicine of Case Western \\ Reserve University, Cleveland, $\mathrm{OH}$ \\ b Department of Nuclear Medicine/Jb3, Cleveland Clinic, Cleveland, $\mathrm{OH}$
}

Received Jun 25, 2020; accepted Jun 25, 2020

doi: $10.1007 / \mathrm{s} 12350-020-02272-4$

\section{See related article, pp. 86-96}

Cardiac imaging is used in patients with established or suspected sarcoidosis for four major purposes: (1) to identify cardiac involvement, (2) to assess severity, extent, location, and stage of disease, (3) to gauge the response to therapeutic intervention, and (4) to aid in stratifying cardiac risk. Active sarcoid granulomas contain inflammatory cells with high glycolytic activity, forming the basis for visualization of the inflammation with fluorine-18 fluorodeoxyglucose (FDG) PET imaging. If normal myocardial glucose uptake is suppressed by diet, prolonged fasting, and IV heparin, then FDG accumulation should reflect glucose use by inflammatory leukocytes. $^{1,2}$

Because visual PET image analysis can be challenging, Miller et $\mathrm{al}^{3}$ sought to examine the benefit of quantitative measurements of perfusion (rubidium-82) and glucose utilization (FDG) for identifying cardiac sarcoidosis (CS). Patients in the study $(n=69)$ ingested a low-carbohydrate, high-fat diet for 36 hours, then fasted for at least 8 hours. Histopathologic confirmation of CS was available in 7 (4 of 5 explanted hearts had CS at transplant, and two endomyocardial biopsies were positive). For the others, the authors applied modified Japanese Ministry of Health and Wellness (JMHW) criteria to identify 29 individuals (42\%) with definite or

Reprint requests: Richard C. Brunken, MD, FAHA, FACC, FASNC, Department of Nuclear Medicine/Jb3, Cleveland Clinic, 9500 Euclid Avenue, Cleveland, OH 44195; rbrunken09@charter.net

J Nucl Cardiol 2022;29:97-100.

1071-3581/\$34.00

Copyright (C) 2020 American Society of Nuclear Cardiology. probable cardiac involvement and who were then considered to have CS.

\section{PET IMAGE ANALYSIS}

Summed rest perfusion scores (SRS) and total perfusion deficit (TPD) scores were obtained from the perfusion images. On the metabolic images, FDG uptake was classified as absent, diffuse, focal, or focal on diffuse. $^{2}$ Myocardial voxels with FDG standardized uptake values $(\mathrm{SUVs})>1.3$ and $>1.5$ above the LV blood pool SUV were identified, yielding VOI's (volumes of interest) of abnormal activity. Cardiometabolic activity (CMA) was derived by multiplying the abnormal cardiac FDG activity volume by mean $\mathrm{SUV}_{\text {max }}$ of the involved tissue $\left(\mathrm{CMA}_{\mathrm{LVBP} 1.3}\right.$ and $\left.\mathrm{CMA}_{\mathrm{LVBP} 1.5}\right)$. Similarly, voxel volumes with $\mathrm{SUV}_{\max }$ values exceeding 2.7 and 4.1 were identified to compute $\mathrm{CMA}_{2.7}$ and $\mathrm{CMA}_{4.1}$. CMA values are measurements which incorporate extent and intensity of abnormal tracer uptake, either adjusted for blood pool activity $\left(\mathrm{CMA}_{\mathrm{LVBP} 1.3}\right.$ and $\left.\mathrm{CMA}_{\mathrm{LVBP} 1.5}\right)$ or above absolute thresholds $\left(\mathrm{CMA}_{2.7}\right.$ and $\left.\mathrm{CMA}_{4.1}\right)$. The study employs a larger population than previous investigations using quantitative methods, ${ }^{4-6}$ and extends prior observations by reporting TPD scores and reproducibility of quantitative FDG measurements.

\section{QUANTITATIVE PET MEASUREMENTS FOR CARDIAC SARCOIDOSIS}

SRS and TPD scores values were significantly higher in those with CS. Accuracy of TPD scores for CS diagnosis was comparable to that of SRS scores (AUC of 0.75 versus 0.67 ), suggesting that quantitation provided little incremental benefit beyond careful visual image analysis. 
Table 1. Abnormal cardiac FDG uptake unrelated to sarcoidosis

\author{
Focal or focal on diffuse uptake \\ Incomplete suppression of normal glucose utilization \\ Infectious myocarditis/endocarditis \\ Pericarditis \\ Papillary muscle uptake (normal variant) \\ Recent ischemic event ("ischemic memory") in CAD \\ Inflammation associated with recent infarction \\ Recent radiofrequency ablation for cardiac arrhythmias, other acute injury \\ Lipomatous hypertrophy of the inter-atrial septum \\ Inflammation associated with aortic stenosis/mitral annular calcification \\ RV uptake due to increased work in pulmonary hypertension \\ Unstable coronary artery plaque/arteritis \\ Lead/device inflammation or infection \\ Artifact due to error in CT attenuation correction for dense device (e.g., AICD lead) \\ Primary/metastatic tumor of the myocardium/pericardium \\ Diffuse FDG uptake \\ Some non-ischemic cardiomyopathies \\ Incomplete suppression of normal myocardial glucose utilization \\ Chemotherapy for malignant tumors
}

On FDG images, a $\mathrm{CMA}_{\mathrm{LVBP} 1.5}$ value $>0$ had the highest diagnostic accuracy (sensitivity $=90 \%$, specificity $=90 \%, \mathrm{NPV}=92 \%, \mathrm{PPV}=87 \%$ with an AUC of 0.92). However, its diagnostic performance did not differ from SUVmax, and both parameters were similar to visual analysis (sensitivity $=83 \%$, specificity $=95 \%$, $\mathrm{NPV}=88 \%, \mathrm{PPV}=92 \%$, AUC 0.89). Abnormal tracer uptake volumes $\left(\mathrm{VOI}_{\mathrm{LVBP} 1.3}, \mathrm{VOI}_{\mathrm{LVBP} 1.5}, \mathrm{VOI}_{2.7}\right.$, and $\mathrm{VOI}_{4.1}$ ) had significantly lower AUC's, suggesting that both intensity and extent of tracer uptake are important for quantitation.

Patient preparation may have influenced the findings. Heparin was not administered and the 8 hour fast is less than that recently suggested. ${ }^{2}$ As such, blood pool spillover to myocardium may have been greater than if heparin or a longer fast had been employed. This may, in part, explain why a parameter like $\mathrm{CMA}_{\mathrm{LVBP} 1.5}$ with compensation for blood pool activity had the best performance. Use of an internal reference value (e.g., blood pool SUV) appears advantageous, in that it may help compensate for inter-patient differences in metabolic milieu that would be difficult to address in clinical practice. Moreover, an internal reference facilitates comparison to images from centers employing different PET/CT instruments or image processing/ analysis software.

\section{DOES FDG QUANTITATION TELL THE FULL STORY?}

While parameters such as $\mathrm{CMA}_{\mathrm{LVBP} 1.5}$ quantitate the extent and severity of FDG uptake and facilitate comparison of serial exams, ${ }^{7}$ they do not account for the spatial distribution of abnormal activity. A patient with a single large intense focus of activity could have a value comparable to an individual with multiple less intense foci of uptake. It is uncertain if both patients would be equally responsive to immunosuppression or have similar cardiac risk. Flores suggested that the location of abnormal FDG uptake may be related to adverse cardiac risk. ${ }^{8}$ Sperry ${ }^{9}$ reported that heterogenous FDG uptake in hypoperfused myocardium, as defined by a greater coefficient of variation, is also associated with a higher adverse risk. More recently, Manabe indicated that "texture analysis", an analysis of the voxel by voxel variability of FDG uptake, is helpful for identifying myocardial involvement by sarcoidosis. ${ }^{10}$ Thus, while the extent and severity of FDG uptake are important descriptors of abnormal tracer accumulation, location and heterogeneity of tracer uptake also appear to provide clinically relevant information.

Miller's study did not integrate the perfusion and FDG information, ${ }^{3}$ and it did not correlate the PET findings with late gadolinium enhancement on MRI. Consideration of both perfusion and metabolism increases the diagnostic accuracy of FDG PET imaging 
for CS. ${ }^{11}$ Moreover, use of perfusion and FDG images has been proposed to stage CS progression. ${ }^{12}$ Early disease is suggested by focally increased FDG uptake without perfusion defect. As CS progresses, abnormal FDG uptake is associated with resting perfusion defect ("perfusion-metabolism mismatch"), which may advance in the end stage to matching perfusion and metabolic defects.

Similarly, others have indicated that the combined utilization of FDG images and late gadolinium enhancement on MRI images benefits CS identification. ${ }^{13,14}$ Quantitation of the extent and severity of abnormal FDG uptake in hypoperfused and normally perfused myocardium, along with the extent and severity of matching defects on PET images (or the degree of late gadolinium enhancement on MRI images) would be clinically relevant for tissue characterization, and for gauging the response to treatment on serial imaging studies.

\section{DO ABNORMAL FDG UPTAKE MEASUREMENTS REFLECT SARCOIDOSIS ACTIVITY?}

In CS patients, the assumption is that abnormal FDG uptake on PET images is reflective of and proportionate to the degree of inflammation. That is, more intense and extensive abnormal FDG uptake is assumed to reflect more inflammation than situations in which tracer uptake is less pronounced and extensive. Moreover, a decrease in the amount of FDG uptake on serial PET studies is considered to reflect a diminution in tissue inflammation, presumably in response to immunosuppressive therapy and/or transition to inactive scar. ${ }^{7,15}$ While reasonable, these assumptions may not necessarily be correct.

First, FDG uptake on cardiac PET images is nonspecific (Table 1) and it does not necessarily equate to inflammation from CS. Inclusion of non-specific FDG uptake into any quantitative measurement will skew the value, resulting in a number indicating greater $\mathrm{CS}$ inflammation than is actually present. As such, a quantitative analysis of FDG uptake should only be performed after a careful review of the patient's clinical data and a focused visual analysis of the fused PET and CT images by an experienced observer. This is necessary to exclude abnormal tracer uptake due to unrelated causes from impacting the analysis for CS inflammatory activity.

Second, FDG uptake is considered to be proportionate to the uptake of unlabeled glucose, with the lumped constant defining the ratio of molecules of FDG accumulated per molecule of glucose. ${ }^{16}$ Glucose typically enters cells employing facilitated transport via GLUT transporters. ${ }^{17}$ Several different GLUT transporters have been identified in white blood cells, lymphocytes and macrophages ${ }^{17}$ and each of these different transporters have differing avidities for glucose, and by inference, FDG. Theoretically, differences in observed FDG uptake from one PET scan to another could result from differences in GLUT transporter expression, despite a similar number of inflammatory cells. The stability of GLUT transporter expression in inflammatory granulomas of sarcoidosis is uncertain and it is unknown if transporter expression is modifiable by glucocorticoids or other immunosuppressive agents.

Finally, uptake of glucose into mammalian cells may also occur via sodium-dependent glucose transporters (SGLTs). FDG is not transported via this type of transporter, and PET FDG images do not trace any glucose which enters tissue via this mechanism. ${ }^{16}$ SGLTs have been identified in the human intestine, kidney and in some cancers (including leukemia). Whether SGLTs are important for glucose uptake in inflammatory granulomas is uncertain; however, if inflammation or immunosuppression resulted in the expression of SGLTs, quantitative PET FDG imaging would underestimate the degree of tissue inflammation.

\section{CONCLUSION}

While tracers depicting somatostatin receptors (gallium-68 dotatate), cell proliferation $\left({ }^{11} \mathrm{C}\right.$-labeled $4^{\prime}$ thiothymidine (4DST) and $3^{\prime}$-deoxy- $3^{\prime}-{ }^{18}$ F-fluorothymidine $\left.\left({ }^{18} \mathrm{~F}-\mathrm{FLT}\right)\right)$, and hypoxia have been explored for visualization of CS with PET, they are not sufficiently validated for routine clinical imaging. FDG is widely available and relatively inexpensive, and clinicians likely will rely upon FDG PET imaging for the diagnosis and follow-up of CS for the foreseeable future. ${ }^{18}$ Multiple factors can contribute to cardiac FDG uptake which is unrelated to $\mathrm{CS}$, resulting in images that are extremely challenging for interpretation. PET images can depict actual tissue tracer concentrations when acquired in an appropriately calibrated scanner, and the FDG studies are well suited for advanced quantitative analyses such as those performed by Miller and colleagues. ${ }^{3}$ However, these quantitative measurements should only be obtained after the images have been visually assessed by an experienced physician. This individual should be familiar with the patient's clinical history and other imaging studies, with in-depth knowledge of the patient-specific factors which could impact FDG accumulation unrelated to sarcoidosis. Moreover, the reader and the referring physician should both be aware of the assumptions made in the use of FDG PET images for identifying and quantifying myocardial inflammation in $\mathrm{CS}$, before using this information to implement therapy and assess treatment response. 


\section{Disclosures}

The author has no conflicts of interest to disclose.

\section{References}

1. Schwartz RG, Malhotra S. Optimizing cardiac sarcoid imaging with FDG PET: Lessons from studies of physiologic regulation of myocardial fuel substrate utilization (editorial). J Nucl Cardiol. 2020;27:490-3.

2. Slart RHJA, Glaudemans AWJM, Lancellotti P, Hyafil F, Blankstein R, Schwartz RG, et al. A joint procedural position statement on imaging in cardiac sarcoidosis: from the Cardiovascular and Inflammation \& Infection Committees of the European Association of Nuclear Medicine, the European Association of Cardiovascular Imaging, and the American Society of Nuclear Cardiology. J Nucl Cardiol. 2018;25:298-319.

3. Miller R, Cadet S, Pournazari P, Pope A, Kransdorf E, Hamilton $\mathrm{M}$, et al. Quantitative assessment of cardiac hypermetabolism and perfusion for diagnosis of cardiac sarcoidosis. J Nucl Cardiol. 2020. https://doi.org/10.1007/s12350-020-02201-5.

4. Ahmadian A, Brogran A, Berman J, Sverdlow AL, Mercier G, Mazzini M, et al. Quantitative interpretation of FDG PET/CT with myocardial perfusion imaging increases diagnostic information in the evaluation of cardiac sarcoidosis. $J$ Nucl Cardiol. 2014;21:925-39.

5. Lebasnier A, Legallois D, Bienvenu B, Bergot E, Desmonts C, Zalcman G, et al. Diagnostic value of quantitative assessment of cardiac ${ }^{18} \mathrm{~F}$-fluoro-2-deoxyglucose uptake in suspected cardiac sarcoidosis. Ann Nucl Med. 2018;32:319-27.

6. Mc Ardle BA, Birnie DH, Klein R, de Kemp RA, Leung E, Renaud J, et al. Is there an association between clinical presentation and the location and extent of myocardial involvement of cardiac sarcoidosis as assessed by ${ }^{18} \mathrm{~F}$-flurodoexyglucose positron emission tomography? Circ Cardiovasc Imaging. 2013;6:617-26.

7. Waller AH, Blankstein R. Quantifying myocardial inflammation using F18-fluorodeoxyglucose positron emission tomography in cardiac sarcoidosis. J Nucl Cardiol. 2014;21:940-3.

8. Flores RJ, Flaherty KR, Jin Z, Bokhari S. The prognostic value of quantitating and localizing F-18 FDG uptake in cardiac sarcoidosis. J Nucl Cardiol. 2018. https://doi.org/10.1007/s12350018-01504-y.
9. Sperry BW, Tamarappoo BK, Oldan JD, Javid O, Culver DA, Brunken R, et al. Prognostic impact of extent, severity, and heterogeneity of abnormalities on ${ }^{18} \mathrm{~F}$-FDG PET scans for suspected cardiac sarcoidosis. J Am Coll Cardiol Imag. 2018;11:33645.

10. Manabe O, Ohira H, Hirata K, Hiyashi S, Naya M, Tsujino I, et al. Use of ${ }^{18} \mathrm{~F}$-FDG PET/CT texture analysis to diagnose cardiac sarcoidosis. Eur J Nucl Med Mol Imaging. 2019;46:1240-7.

11. Kim SJ, Pak K, Kim K. Diagnostic performance of F-18 FDG PET for detection of cardiac sarcoidosis; A systematic review and meta-analysis. J Nucl Cardiol. 2019. https://doi.org/10.1007/s123 50-018-01582-y.

12. Blankstein R, Waller AH. Evaluation of known or suspected cardiac sarcoidosis. Circ Cardiovasc Imaging. 2016;9:e000867. h ttps://doi.org/10.1161/CIRCIMAGING.113.000867.

13. Dweck MR, Abgral R, Trivieri MG, Robson PM, Karakatsanis N, Mani V, et al. Hybrid magnetic resonance imaging and positron emission tomography with fluorodeoxyglucose to diagnose active cardiac sarcoidosis. J Am Coll Cardiol Imaging. 2018;11:94-107.

14. Vita T, Okada DR, Veillet-Chowdhury M, Bravo PE, Mullins E, Hulten E, et al. Complementary value of cardiac magnetic resonance imaging and positron emission tomography/computed tomography in the assessment of cardiac sarcoidosis. Circ Cardiovasc Imaging. 2018. https://doi.org/10.1161/CIRCIMAGING. 117.007030.

15. Ahmadian A, Pawar S, Govender P, Berman J, Ruberg FL, Miller EJ. The response of FDG uptake to immunosuppressive treatment on FDG PET/CT imaging for cardiac sarcoidosis. J Nucl Cardiol. 2016;24(2):413-24.

16. Barrio JR, Huang SC, Satyamurthy N, Scafoglio CS, Yu AS, Alavi A, Krohn K. Does 2-FDG PET accurately reflect quantitative in vivo glucose utilization? J Nucl Med. 2020;61:931-7.

17. Vrhovac I, Breljak D, Sabolic I. Glucose transporters in the mammalian blood cells. Periodicum Biologorum. 2014;116:131-8.

18. Chareonthaitawee P, Beanlands RS, Chen W, Dorbala S, Miller EJ, Murthy VL, et al. Joint SNMMI-ASNC expert consensus document on the role of ${ }^{18} \mathrm{~F}$-FDG PET/CT in cardiac sarcoid detection and therapy monitoring. J Nucl Cardiol. 2017;24:174158.

Publisher's Note Springer Nature remains neutral with regard to jurisdictional claims in published maps and institutional affiliations. 